\title{
Une nouvelle approche de la stabilité des remblais sur sols mous traités par colonnes ballastées
}

\section{A. DHOUIB}

Responsable des études géotechniques à Solen études, 50 , rue Eugénie-Le-Gullermic, 94290 Villeneuve-le-Roi

\section{M.P. GAMBIN \\ 21, quai d'Anjou, 75004 Paris}

\section{S. JACQUEMIN}

Ingénieur ENSG de Nancy, Ex-ingénieur stagiaire à la SNCF-VP, résidence Allindor-Montebello, 97170 Petit-Bourg

\section{B. SOYEZ}

LCPC Paris, 58, boulevard Lefebvre, 75732 Paris Cedex 15
On présente une nouvelle méthode d'analyse de stabilité des ouvrages en terre sur sols mous traités par colonnes ballastées. On formule les résistances au cisaillement des sols pour trois procédures de modélisations différentes : modèle initial (début du chargement), homogénéisation de Priebe et modèle réel (correspondant à l'état final de transfert de charge). On compare les trois modèles en présentant, en rupture circulaire de Bishop, l'évolution théorique du coefficient de sécurité vis-à-vis du glissement généralisé $\Gamma_{\min }$ en calcul aux états limites ultimes (ELU) exprimé en fonction du taux d'incorporation a.

Grâce à une méthode élaborée matérialisant la concentration des contraintes à la verticale des colonnes à l'interface remblai-sol, on construit, dans le cadre du modèle réel, des abaques de la variation du coefficient de sécurité en fonction du pourcentage d'incorporation pour divers taux de chargement et pour un angle de frottement du ballast égal soit à $38^{\circ}$ soit à $42^{\circ}$. Enfin, on démontre l'incidence de la variation des paramètres (caractéristiques de cisaillement du remblai et des colonnes, rapport de concentration des contraintes et taux de chargement) sur l'évolution de $T_{\min }$.

\section{A new approach of embankment stability on soft soils reinforced by stone columns}

A new analysis of embankment stability on soft soil reinforced by stone columns in presented.

The theoretical shear strength and minimum safety factors $\mathrm{T}$ obtained along Bishop circular slip surface for the initial model (beginning of the first loading), the Priebe equivalent model and the real model based on stress concentration on columns are compared. This involves the use of a complex method for generalising stress concentration at the interface of the embankment and the improved soil on the columns top. Charts presenting minimum safety factors $\Gamma_{\text {mi }}$ versus replacement ratio a are given for friction angle of stone $\varphi$, varying between $38^{\circ}$ and $42^{\circ}$.

The parametric study concerning the effect of shearing resistance values of the embankment (in terms of friction angle $\varphi$ ) and of the column (in terms of cohesion c), the stress concentration ratio $n$ and the loading level $\sigma_{t} / \mathrm{c}_{\mathrm{u}}$ on $\Gamma_{\min }$ completes this study. 
taux d'incorporation (ou coefficient de substitution).

A : section droite totale du domaine d'influence de la colonne (principe de la cellule unitaire).

Ac : section droite de la colonne.

$c_{\mathrm{c}}$ : cohésion de la colonne.

$c_{e} \quad$ : cohésion équivalente du milieu homogénéisé.

c. : cohésion du remblai $(=0)$.

$c_{\mathrm{u}} \quad$ : cohésion non drainée du sol mou.

$c_{u o}$ : résistance au cisaillement non drainée initiale du sol mou.

h. : hauteur maximale du remblai.

k : coefficient multiplicateur.

m : facteur d'homogénéisation courant.

$\mathrm{m}_{\min }$ : facteur d'homogénéisation minimal $(=\mathrm{a})$.

$\mathrm{m}_{\operatorname{mix}}$ : facteur d'homogénéisation maximal.

$m_{c}$ : taux de contribution de la colonne $\left(=m_{\max }\right)$.

$\mathrm{m}_{\mathrm{s}}$ : taux de contribution du sol.

n : rapport de concentration des contraintes,

r : rapport des contraintes de cisaillement du milieu réel au milieu homogénéisé.

$x \quad$ : variable de la dimension horizontale.

$t_{i} \quad$ : temps initial $(=0$, début du chargement),

$t_{\mathrm{f}} \quad$ : temps final de transfert de charge.

$\alpha_{\mathrm{s}} \quad$ : angle d'inclinaison de la surface potentielle de rupture dans le sol en un point.

$\alpha \quad$ : angle d'inclinaison de la surface potentielle de rupture dans la colonne en un point.

$\gamma_{c} \quad$ : poids volumique du matériau de la colonne.

$\gamma_{\text {r }}$ : poids volumique du remblai.

$\gamma_{1}$ : poids volumique du sol mou.

$\gamma_{f} \quad$ : poids volumique équivalent du milieu homogenéisé.

$\Gamma_{c 4} \quad$ : coefficient de sécuritê partiel sur la cohésion non drainée du sol mou.

$\Gamma_{e}:$ coefficient de méthode (d'homogénéisation).

$\Gamma_{a \text { h }} \quad$ : coefficient de sécurité partiel sur l'étreinte latérale (DTU 13-2).
$\Gamma_{\min } \quad$ : coefficient de sécurité minimal obtenu par la méthode de rupture circulaire de Bishop.

$\Gamma_{s_{3}}$ : coefficient de méthode de calcul de stabilité (Bishop).

$\Gamma_{\text {or }} \quad$ : coefficient de sécurité partiel sur l'angle de frottement du remblaí,

$\Gamma_{\varphi c} \quad$ : coefficient de sécurité partiel sur l'angle de frottement de la colonne.

$\Delta c_{u} \quad$ : accroissement de la cohésion non drainée du sol.

$\varphi_{c}^{\prime} \quad$ : angle de frottement intergranulaire du ballast $\left(\varphi_{c}^{\prime}=\varphi\right)$.

$\varphi_{c u} \quad$ : angle de frottement du sol mou en conditions non drainées $\left(\varphi_{c u}=0\right)$.

$\varphi$, : angle de frottement du remblai.

$\sigma_{c} \quad$ : contrainte verticale appliquée à la base du remblai sur la colonne.

$\sigma \quad$ : contrainte verticale équivalente mobilisée dans le milieu homogénéisé à la profondeur $z$.

$\sigma_{f} \quad$ : contrainte verticale moyenne apportée par le remblai de chargement.

$\sigma \quad$ : contrainte verticale appliquée à la base du remblai sur le sol mou.

$\sigma_{\text {st }}^{\prime} \quad$ contrainte verticale effective régnant dans la colonne à la profondeur $\mathrm{z}$.

$\sigma_{\text {vclim }}$ : contrainte verticale effective de rupture de la colonne à la profondeur $\mathrm{z}$.

$\sigma_{\text {hmix }}^{\prime}$ : contrainte maximale effective que peut supporter le sol autour de la colonne.

$\sigma_{\text {ho }}$ : contrainte effective horizontale régnant dans le sol avant traitement.

$\tau_{c} \quad$ : contrainte de cisaillement mobilisée dans la colonne.

$\tau_{\infty e} \quad$ : contrainte de cisaillement mobilisée dans la colonne à l'état initial $\left(\mathrm{t}_{1}=0\right)$.

$\tau$. : contrainte de císaillement mobilisée dans le milieu homogénéisé.

$\tau_{\mathrm{s}} \quad$ : contrainte de cisaillement mobilisée dans le sol mou.

$\tau_{x o} \quad$ : contrainte de cisaillement mobilisée dans le sol à l'état initial $(\mathrm{t}=0)$.

\section{1}

\section{Introduction}

Les études de dimensionnement des colonnes verticales frottantes sous remblais, radiers et semelles sont relativement nombreuses. Plusieurs auteurs (Priebe, 1976 ; Goughnour et Bayuk, 1979 ; Balaam et Poulos, 1983; Van Impe et De Beer, 1983) proposent des graphiques permettant de calculer le rapport de concentration des contraintes $\mathrm{n}$ en fonction du taux d'incorporation a. Sous les remblais suffisamment longs ou les radiers de grandes dimensions, les tassements peuvent être, selon les cas de charge, estimés à partir des abaques de Priebe (1976) ou de Van Impe et De Beer
(1983), voire de Balaam et al. (1977) pour des fondations rigides.

En France, les documents techniques unifiés (DTU) 13-2 proposent, par analogie avec l'essai triaxial, de lier la contrainte verticale de rupture de la colonne à l'étreinte latérale du sol multipliée par le coefficient de butée du matériau de traitement. Pour les projets « sensibles m, on recourt souvent à une analyse numérique par éléments finis permettant de déterminer, au préalable, les champs des déplacements et des contraintes dans les colonnes et dans le sol. Après édification de l'ouvrage, il est procédé à la mise en place d'instruments de suivi et de mesures des tassements, des contraintes et de la dissipation des pressions interstitielles (Liausu et Juillié, 1990). 
Les calculs de stabilité des talus limitant ces remblais vis-à-vis du grand glissement sont couramment conduits par une approche d'homogénéisation de type Priebe (1978). Cette approche est simple et fort intéressante. Mais, nous avons démontré (Dhouib et al., 1993) les divergences des résultats de calculs de stabilité des remblais sur sols mous traités par colonnes ballastées en utilisant le modèle initial (début du chargement), I'homogénéisation de Priebe (1978) et le modèle réel (état final du report de charge).

En effet, la formulation de la résistance au cisaillement mobilisée le long d'une surface de rupture potentielle selon chaque type de sols permet de montrer l'incidence du mécanisme de transfert de charge sur l'amélioration de la stabilité de ces ouvrages. La démarche proposée ici consiste à étudier le modèle initial, le modèle homogénéisé de type Priebe (1978) et le modèle réel. Tous calculs faits (Dhouib et al., 1997a), des abaques destinés à la recherche du coefficient de sécurité global sont élaborés en fonction du taux d'incorporation dans le cadre du modèle réel.

\section{2}

\section{Comportement des ouvrages en terre sur sols mous traités par colonnes ballastées}

\section{1}

\section{Position du problème - Constatations expérimentales}

Trois exemples de remblais sur sols mous traités par colonnes ballastées seront considérés (Fig. 1) :

- remblais d'accès au pont Mathilde pour franchir la Seine en amont de Rouen (Vautrain, 1980) ;

- chargement en vraie grandeur d'argile déposée par voie hydraulique en bord de Seine (Soyez et al., 1983) :

- étude du comportement d'une culée en terre armée fondée sur alluvions argileuses hétérogènes pour le franchissement de la Sarre, (Iorio et al., 1987).

La figure 1a montre les étapes de construction des trois remblais et la figure 1 b l'évolution en fonction du temps du rapport de concentration des contraintes n.

L'examen de l'évolution de n en fonction du temps conduit à distinguer (Fig. 2):

1) L'état initial : au début du chargement ( $t=t=0)$, il n'y a pas de report de charge sur les colonnes, donc il n'y a pas de réduction de la contrainte sur le sol. Les colonnes et le sol sont soumis à la contrainte $\sigma_{+}$apportée par les remblais lors de l'étape initiale de chargement. On définit ainsi un modèle initial qui peut correspondre à un rapport de concentration des contraintes $n=1$ (Fig. 2a).

2) L'état de transfert progressif de charge : après un temps relativement court (mais difficile à estimer dans les trois cas présentés sur la figure 1), on observe un report progressif de charge qui provoque, entre le temps initial $t$ et un certain temps final $t_{r}$ une augmentation de la contrainte verticale $(\sigma)$ sur la colonne et une diminution de la contrainte verticale $(\sigma)$ sur le sol. Le rapport de concentration $n$ augmente en fonction du temps
Tomps (jours)

$\begin{array}{lllllllllllll}0 & 20 & 40 & 60 & 80 & 100 & 120 & 140 & 160 & 180 & 200 & 220\end{array}$
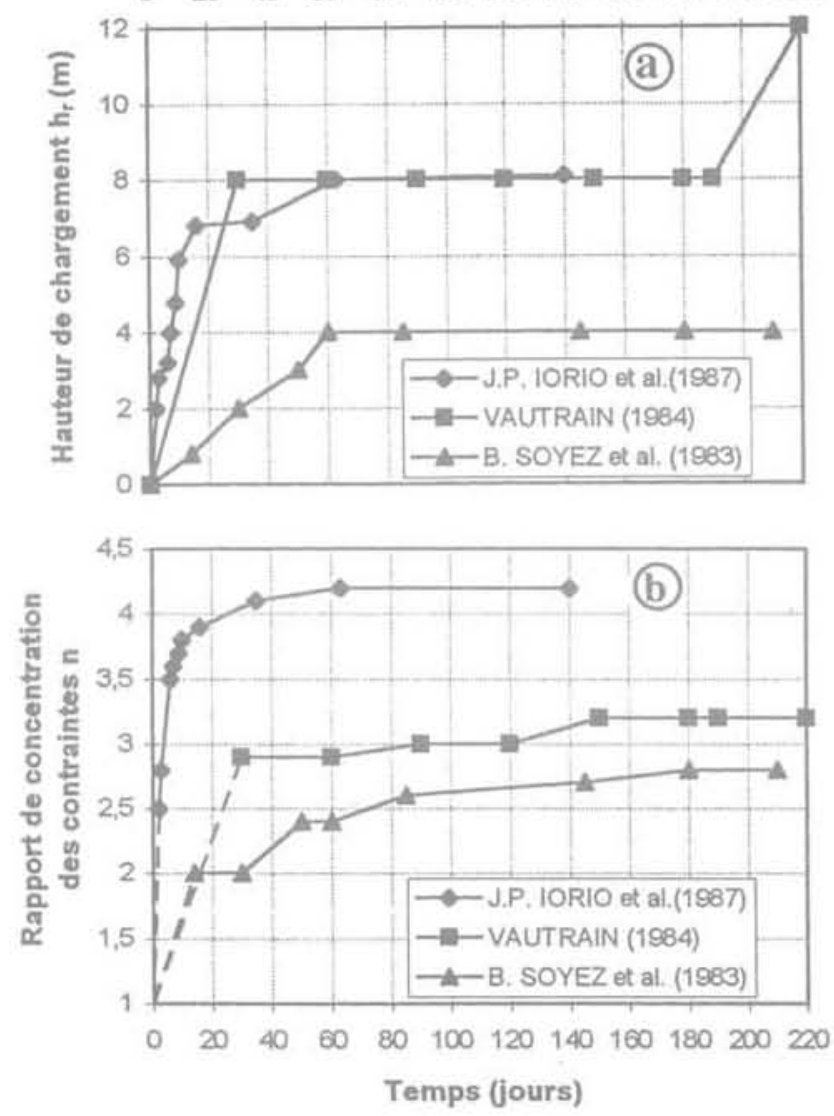

FG.1 Reports de contraintes observés sur trois chantiers de remblais sur sols mous traités par colonnes frottantes.

Full scale loading by embankments on soft soils. improved by stone columns, transfer as observed.

jusqu'à une valeur maximalen $n_{\text {max }}$ Dans cette phase, on peut poser que $n=\alpha \cdot n$.max $_{\text {avec }}$ av $<\alpha \leq 1$ (Fig. 2b).

3) L'état final de transfert de charge : à partir d'un certain temps final t, compris dans les trois cas de la figure 1 entre 30 et 80 jours, il y a stabilisation des contraintes sur les colonnes et sur le sol. On définit le modèle réel qui correspond à l'état final de transfert de charge oủ $n=n_{\max }$ (Fig. $2 \mathrm{c}$ ).

Le modèle initial et le modèle réel seront comparés par la suite à l'homogénéisation de Priebe.

\section{2}

\section{Formulation de la résistance au cisaillement}

Considérons les trois modèles (modèle initial et modèle réel définis ci-dessus et homogénéisation de Priebe) avec les surfaces de rupture potentielles correspondantes de la figure 3 . On suppose que le niveau de la nappe phréatique est confondue avec le niveau du sol initial, ce qui est souvent le cas.

\section{Modèle initial}

Dans le modèle initial de la figure $3 a$, la résistance au cisaillement mobilisée en un point de la 

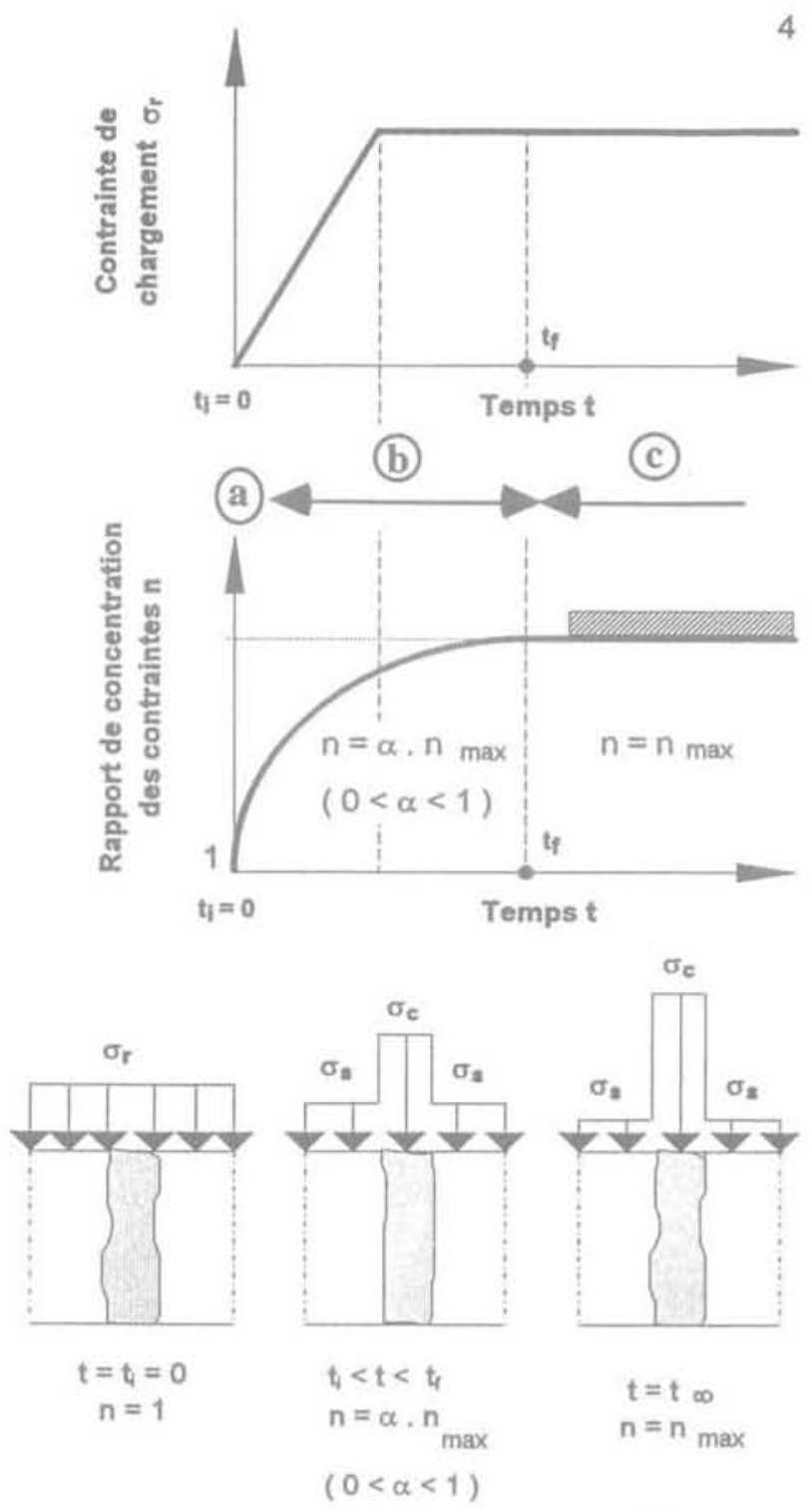

Etat initial

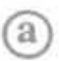

Etat

final de charge

Avec :

teuses mais plus complexes, c'est la procédure d'homogénéisation de Priebe (1978) qui reste la plus utilisée en pratique. Elle consiste à transformer le modèle initial (remblai + sol et colonnes de la figure 3a) en un modèle équivalent composé du même remblai et d'un sol d'assise homogénéisé (Fig. $3 \mathrm{~b}$ ). Ce qui revient à conférer à ce dernier les paramètres équivalents suivants :

$$
\begin{aligned}
\gamma_{c} & =a \cdot \gamma_{c}+(1-a) \cdot \gamma_{s} \\
c_{e} & =(1-m) \cdot c_{u} \\
\operatorname{tg} \varphi_{e} & =m \cdot \operatorname{tg} \varphi_{c}
\end{aligned}
$$

- a le pourcentage d'incorporation, égal au rapport de la section droite de la colonne ballastée (A) à l'aire totale $(A)$ du domaine d'influence de la colonne (principe de la cellule unitaire), soit :

$$
\mathrm{a}=\frac{\mathrm{A}_{\mathrm{c}}}{\mathrm{A}}
$$

- m facteur d'homogénéisation donné par:

$\mathrm{m}_{\min }<\mathrm{m}<\mathrm{m}_{\max }$ (Priebe, 1978; Mitchell, 1981),

où $m_{\min }=a$ et $m_{\max }=a \cdot \frac{\sigma_{c}}{\sigma_{f}}$;

$-\mathrm{c}_{\mathrm{u}}=\mathrm{c}_{\mathrm{uo}}+\Delta \mathrm{c}_{\mathrm{u}}\left(\Delta \mathrm{c}_{\mathrm{u}}\right.$ désigne l'accroissement de la résistance au cisaillement non drainé du sol sous $\sigma_{s}$ ).

La résistance au cisaillement mobilisée en un point de la surface de rupture potentielle est :

$$
\tau_{e}(x)=c_{e}+\sigma_{e}(x) \cdot \cos \alpha \cdot \operatorname{tg} \varphi_{e}
$$

où $\sigma_{e}(\mathrm{x})$ désigne la contrainte verticale issue de la procédure d'équivalence et donnée par:

$$
\sigma_{e}(x)=\sigma_{r}(x)+\gamma_{e} \cdot z
$$

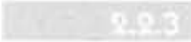 \\ Modèle réel (état final de transfert de charge)}

La charge verticale appliquée initialement au sol mou $\left(\sigma_{r}\right)$ se répartit entre les colonnes $(\sigma)$ et le sol $(\sigma)$ en proportion de leurs aires respectives (Fig. 3c). On peut écrire :

$$
\sigma_{\mathrm{r}}=\mathrm{a} \cdot \sigma_{\mathrm{c}}(\mathrm{x})+(1-\mathrm{a}) \cdot \sigma_{\mathrm{s}}(\mathrm{x})
$$

Le rapport de concentration des contraintes verticales n est défini par :

$$
\mathrm{n}=\frac{\sigma_{\mathrm{c}}(\mathrm{x})}{\sigma_{\mathrm{s}}(\mathrm{x})}
$$

L'état final de transfert de charge conduit à (Aboshi et al., 1979) :

$$
\begin{array}{lr}
\tau_{s}(\mathrm{x})=\mathrm{c}_{\mathrm{u}} & \text { (dans le sol) } \\
\tau_{\mathrm{c}}(\mathrm{x})=\sigma_{\mathrm{vc}}^{\prime}(\mathrm{x}) \cdot \cos \alpha_{\mathrm{c}} \cdot\left(\operatorname{tg} \varphi^{\prime}\right) & \text { (dans la colonne) }
\end{array}
$$

$$
\tau_{\text {so }}(x)=c_{\text {uo }}
$$

dans le sol avec $\mathrm{c}_{\mathrm{uo}}$ résistance au cisaillement non drainée initiale du sol ;

$$
\tau_{\mathrm{co}}(\mathrm{x})=\left\{\sigma_{\mathrm{p}}(\mathrm{x})+\gamma_{\mathrm{c}} \cdot \mathrm{z}\right] \cdot \cos \alpha_{\mathrm{c}} \cdot\left(\operatorname{tg} \varphi_{\mathrm{c}}\right)
$$

dans la colonne, le ballast étant déjaugé :

$$
\text { où : } \sigma_{r}(x)=\gamma_{r} \cdot h_{r}
$$

$\mathrm{h}_{\mathrm{r}}$ étant la hauteur maximale du remblai.

\section{0}

\section{Homogénéisation de Priebe}

Bien que plusieurs auteurs (de Buhan et Salençon, 1987 ; Pruchnicki et Shahrour, 1991) aient élaboré récemment des procédures d'homogénéisation plus promet-

En admettant que la colonne est en état de rupture triaxiale (DTU 13-2), la contrainte verticale effective de rupture de la colonne $\sigma_{\text {vclim }}^{\prime}$ s'écrit (Greenwood, 1970) :

$$
\sigma_{\mathrm{vclim}}^{\prime}=\tan ^{2}\left(\frac{\pi}{4}+\frac{\varphi_{c}}{2}\right) \cdot \sigma_{\text {hmax }}^{\prime}
$$

où $\sigma_{\text {hmax }}^{\prime}$ désigne la contrainte horizontale effective maximale radiale que le sol peut supporter autour de la colonne et qui peut se mettre sous la forme générale suivante (Soyez, 1985) :

$$
\sigma_{\text {hmax }}^{\prime}=\sigma_{h o}^{\prime}+k \cdot c_{u}
$$



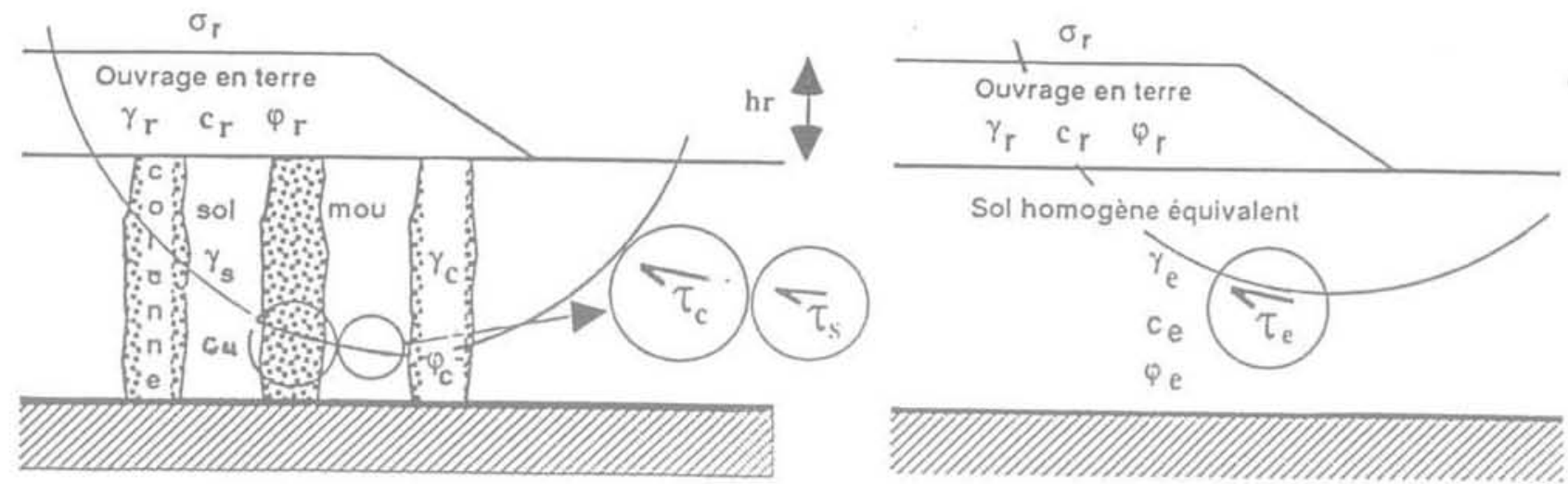

Sol homogène équivalent

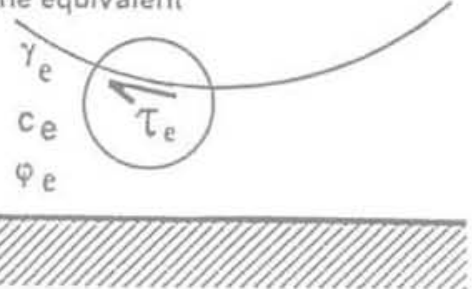

a- Modèle initial

Initial model b- Homogénéisation de PRIEBE (1978) PRIEBE homogeneized model (1978).

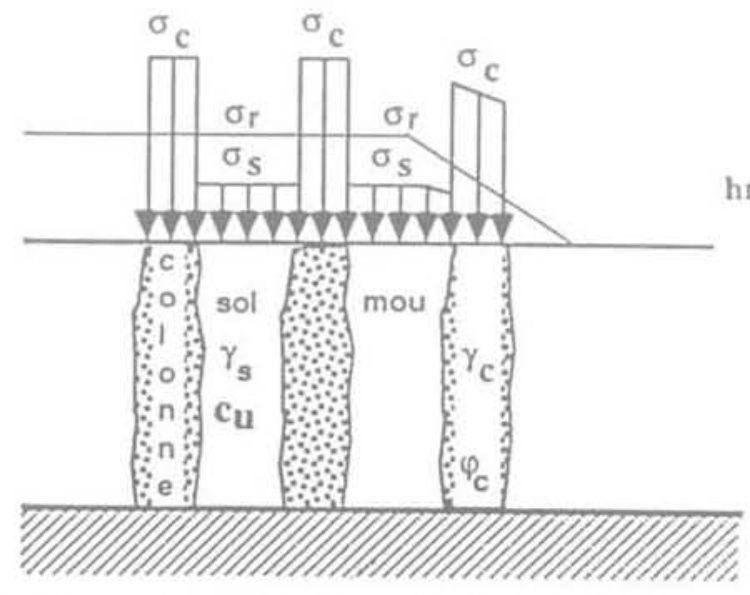

c- Mécanisme de transfert de charge Stress concentration mechanism.

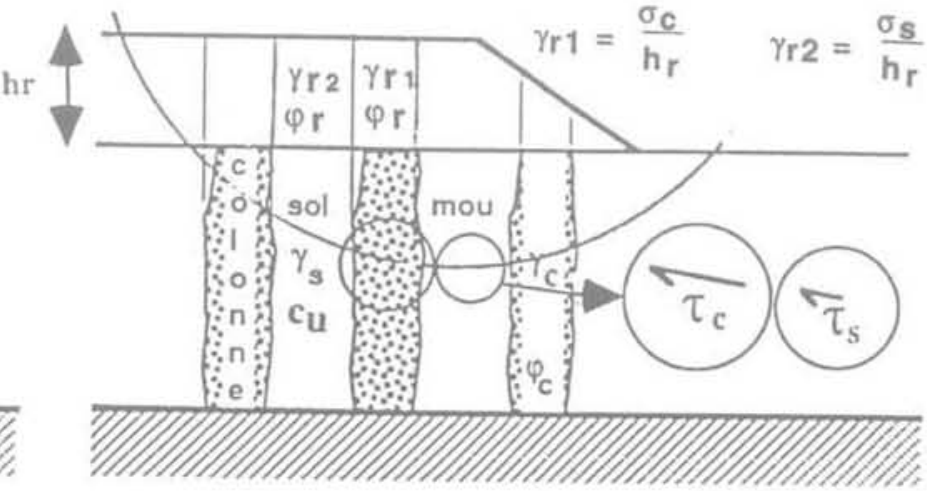

d. Modèle réel (report de charge)

Real model (loading report).

FG.3 Différentes modélisations étudiées sous charges monotones.

a. Modèle initial.

b. Homogénéisation de Priebe (1978).

c. Mécanisme de transfert de charge.

d. Modèle réel (report de charge).

Various modelings under monotonic loadings.

a. Initial model.

b. Priebe homogeneized model (1978)

c. Stress concentration mechanism.

d. Real model (loading report).

avec :

$\sigma_{\text {ho }}$ : contrainte horizontale effective régnant dans le sol avant traitement :

$\mathrm{k}=$ coefficient multiplicateur (Soyez, 1985) qui dépend de la cohésion non drainée $c_{\text {. }}$ et du module d'élasticité $E_{s}$ (Brauns, 1978) ainsi que du coefficient de Poisson $v_{s}$ du sol (Hugues et Withers, 1974) et dont la valeur conseillée en pratique courante est de 4 (Soyez, 1985).

Cette formulation (équation 3.b) suppose que la pression interstitielle $u$ à la périphérie de la colonne est identique à la pression hydrostatique u existant dans le sol avant traitement (Ghionna et Jamiolkowski, 1981; Smoltczyk, 1983).

Si des essais pressiométriques Ménard ont été réalisés dans le sol mou, on peut aussi écrire:

$$
\sigma_{\text {tminax }}^{\prime}=p_{i}-u
$$

où $\mathrm{p}_{1}$ désigne la pression limite conventionnelle. $\mathrm{A}$ noter que, si l'on adopte $\mathrm{p}_{1}-\sigma_{\mathrm{hn}}^{\prime}=5,5 \mathrm{c}_{u}$ pour $\mathrm{p}_{1}<300 \mathrm{kPa}$, cela revient à adopter $\mathrm{k}=5,5$.

\section{3}

\section{Analyse des résistances au cisaillement - Étude comparative}

\section{anasit \\ Rapports des résistances au cisaillement}

On examine les rapports des résistances au cisaillement mobilisées le long de la surface de rupture potentielle. Par souci de simplification, on considère que :

- le ballast $\left(\varphi_{\mathrm{c}}=\varphi^{\prime}\right)$ et le sol mou $\left(c_{\mathrm{u}^{\prime}}, \varphi_{\mathrm{cu}}=0\right)$ sont tous deux non pesants;

- les surfaces de rupture potentielles possèdent, pour deux tranches verticales voisines dans le sol et dans la colonne, les mêmes angles d'inclinaison $\alpha_{c}=$ $\alpha=\alpha$ ) par rapport à l'horizontale, ce qui permet de procéder à des simplifications dans l'expression des rapports de résistance au cisaillement. On voit ainsi que : 
- le report de charge conduit à l'amélioration de la contrainte au cisaillement qui vaut :

$\Delta c_{u}$ dans le sol et $\left(\sigma_{c}-\sigma_{f}\right) \cdot \cos \alpha_{c} \cdot\left(\operatorname{tg} \varphi_{c}^{\prime}\right)$ dans la colonne;

- l'état final de transfert de charge comparé au modèle homogénéisé de Priebe conduit aux rapports des résistances au cisaillement $r_{s}$ et $r_{c}$ suivants :

$r_{s}=\frac{\tau_{s}(x)}{\tau_{e}(x)}=\frac{1}{m_{s}+\left(1-m_{s}\right) \cdot \frac{\sigma_{r}(x)}{c_{u}} \cdot \cos \alpha \cdot\left(\operatorname{tg} \varphi_{c}^{\prime}\right)}$
$r_{c}=\frac{\tau_{c}(x)}{\tau_{e}(x)}=r_{s} \cdot \frac{m_{c}}{a} \cdot \frac{\sigma_{f}(x)}{c_{u}} \cdot \cos \alpha \cdot\left(\operatorname{tg} \varphi_{c}^{\prime}\right)$

avec: $\left\{\begin{array}{l}m_{c}=a \cdot \frac{\sigma_{c}(x)}{\sigma_{r}(x)} \\ m_{s}=(1-a) \cdot \frac{\sigma_{s}(x)}{\sigma_{f}(x)}\end{array}\right.$

(colonne)

dont la somme est égale à l'unité et que nous appelons " taux de contribution des colonnes et du sol ». On désigne par $r$ le rapport des contraintes de cisaillement de l'état final de transfert de charge au milieu homogénéisé de Priebe, soit :

$$
\mathrm{r}=\mathrm{a} \cdot \mathrm{r}_{\mathrm{c}}+(1-\mathrm{a}) \cdot \mathrm{r}_{\mathrm{s}}
$$

Dans l'étude comparative qui suit, l'analyse de r en fonction de a est abordée en calcul aux états limites ultimes (ELU) et en calcul traditionnel (Recommandations CLOUTERRE, 1991).

\section{9ing \\ Étude comparative : modèle réel et homogénéisation de Priebe}

Le rapport des contraintes de cisaillement $\mathrm{r}$ du milieu réel au milieu homogénéisé est analysé en calcul aux ELU (Fig. 4a) et en calcul traditionnel (Fig. 4b). En calcul aux ELU, on adopte

- un coefficient de méthode $\Gamma_{\text {. }}$ pris sommairement égal à 1,125 pour tenir compte, dans le calcul des paramètres équivalents (équa. 1), des approximations inhérentes à la méthode d'homogénéisation

- des coefficients de sécurité partiels sur les para. mètres de cisaillement des sols en combinaisons fon-
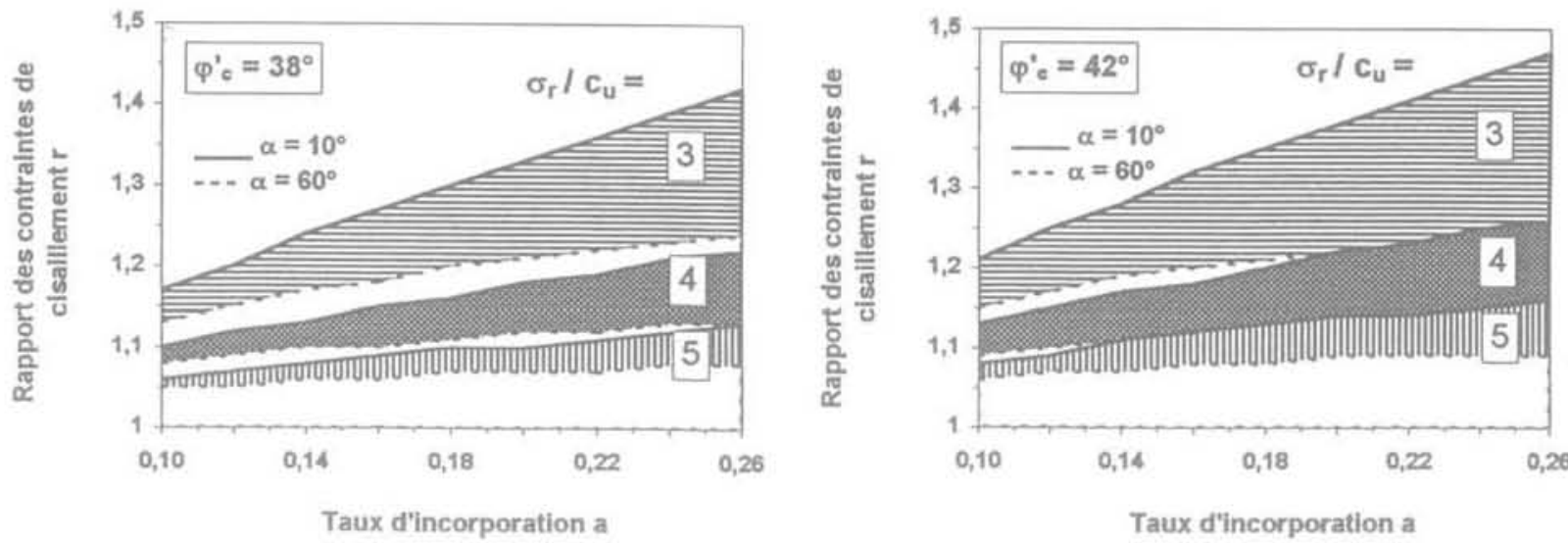

a - Calcul aux États Limites Ultimes (ELU) : $\Gamma_{\dot{c} u}=1,40, \Gamma_{\varphi c}=1,20$ et $\Gamma_{\dot{e}}=1,125$
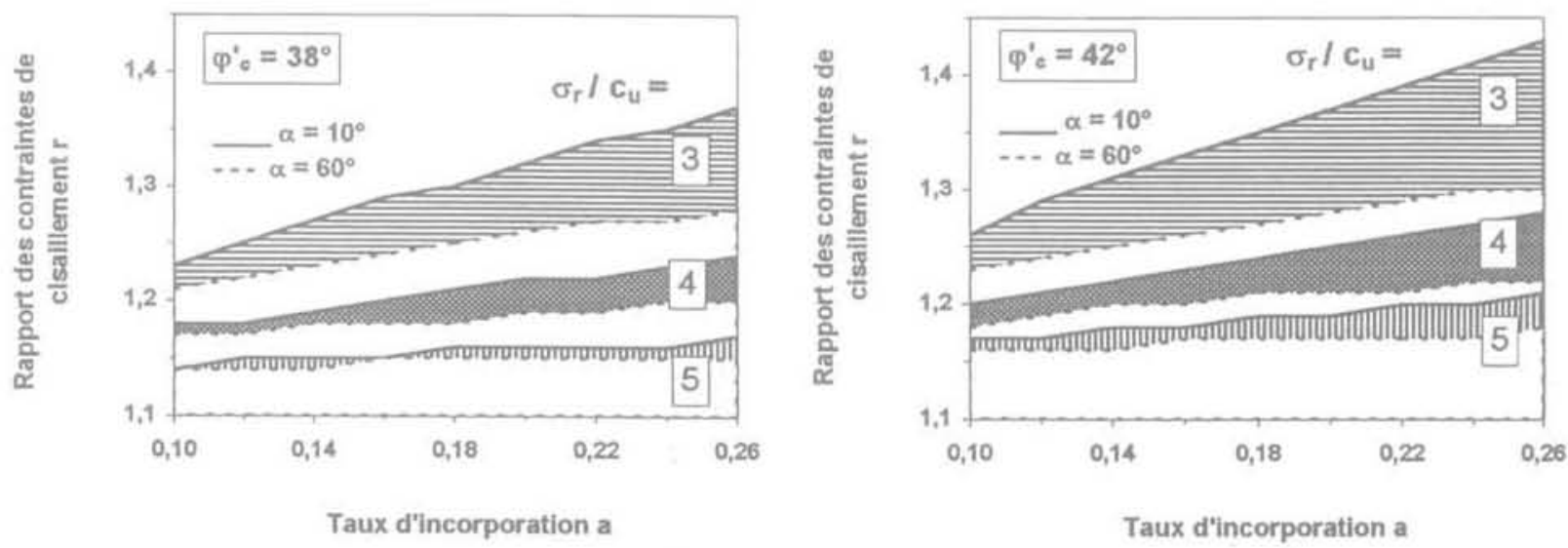

b - Calcul traditionnel avec $\Gamma_{\mathrm{Cu}}=\Gamma_{\varphi \mathrm{C}}=\Gamma_{\dot{\mathrm{e}}}=1,00$.

Taux d'incorporation a

FG.4 Rapport des résistances de cisaillement $\mathrm{r}$ en fonction de a (modèle réel/homogénéisation de Priebe, 1978).

Shear strength ratio $r$ versus area ratio a : effect of stress concentration compared to the equivalent model. Priebe (1978).

a. Calcul aux états limites ultimes (ELU) : $\Gamma_{f u}=1,40, \Gamma_{f c}=1,20$ et $\Gamma_{c}=1,125$.

b. Calcul traditionnel avec: $\Gamma_{\mathrm{cu}}=\Gamma_{\mathrm{ec}}=\Gamma_{\mathrm{e}}=1,00$. 
damentales (Recommandations CLOUTERRE, 1991) $\Gamma_{\mathrm{cu}}$ de 1,40 (sol sensible), $\Gamma_{e c}$ de 1,20 (ballast courant).

Le calcul des paramètres intervenant dans l'égalité (4) est effectué selon les étapes suivantes :

a) Pour un taux de chargement $\sigma / \mathrm{c}_{u}$ donné, la valeur de $\sigma$ est calculée à partir des équations (3) pour un coefficient de sécurité partiel $\Gamma_{\text {ah }}$ de 2 (DTU 13-2) et pour $k=4$ (Soyez, 1985), en négligeant l'accroissement de la cohésion non drainée du sol $\Delta c_{4}$ $\left(c_{u}=c_{u j}\right)$. Cette hypothèse repose sur le fait que $\Delta c_{u}$ est faible sous la contrainte $\sigma_{c}$. En plus, son calcul est délicat.

b) En faisant varier les valeurs de a $(=0,10$ à 0,26$)$, on calcule à partir de l'égalité (2) la valeur de $\sigma$, pour déduire les taux de contribution de la colonne $m_{\varepsilon}$ et du sol $\mathrm{m}_{\mathrm{a}}$.

Il ressort des graphiques de la figure 4 cque la distribution linéaire de $\mathrm{r}$ en fonction de a conduit, pour $\varphi_{c}^{\prime}=38^{\circ}($ Schulze, 1978$)$ et $42^{\circ}$, à des valeurs qui sont toutes supérieures à l'unité. En plus, r décroît pour des rapports de $\sigma / \mathrm{c}_{\mathrm{u}}$ croissants avec une quasi-stabilisation pour $\sigma_{/} / \mathrm{c}_{\mathrm{u}}$ élevé. Ce résultat s'explique par le fait que, lorsque $\sigma / c_{i}$ augmente, la contribution de la colonne est moins importante que celle du sol (cohésion faible et chargement élevé).

La comparaison avec l'homogénéisation de Priebe montre que l'état final de transfert de charge conduit à une nette augmentation de la résistance de cisaillement au sein de la colonne et donc à l'amélioration de la stabilité vis-à-vis du glissement qui fera l'objet du chapitre suivant.

\section{3}

\section{Analyse de stabilité - Construction et comparaison des modèles}

L'analyse de stabilité consiste à chercher le coefficient de sécurité minimal noté $\Gamma_{\min }$ (Recommandations CLOUTERRE, 1991) pour les cercles de rupture potentiels. Les calculs sont conduits par le logiciel de calcul à la rupture «Talren » (F. Blondeau et al., 1984) par la méthode des tranches circulaires de Bishop. Après une étude de l'incidence du nombre des tranches sur les valeurs de $\Gamma_{\min }$, les calculs sont effectués avec 49 tranches verticales, soit en moyenne 2 à 5 tranches verticales par colonne.

\section{1}

\section{Choix des paramètres de calcul et construction des modèles}

\subsection{1}

\section{Sols et paramètres de calcul}

Les différents sols et les paramètres de chaque modèle sont résumés dans le tableau ci-dessous :

\section{Remarques}

1) Le modèle initial comprend trois sols distincts : remblai, sol mou de résistance au cisaillement non drainée initiale ( $c_{u}$ ) et colonnes ballastées (Fig. 3a) alors que le modèle de Priebe comprend uniquement deux sols : même remblai et sol homogénéisé (Fig. 3b) dont les paramètres équivalents sont déduits des équations (1). Pour ces deux modèles, les calculs de stabilité seront effectués en considérant la charge $(\sigma)$ apportée par le remblai sur sa base.

2) Le modèle réel est conçu en assimilant le remblai à un massif de sol caractérisé par un frottement intergranulaire homogène $\varphi_{r}$ (et éventuellement dè la cohésion c) et aussi par un poids volumique moyen variable localement en fonction de la répartition réelle des contraintes à la base du remblai (Fig. 3c). Ce modèle comporte quatre sols distincts : sol mou, colonnes ballastées et remblai composé de deux sols de poids volumiques différents $\gamma_{r 1}$ et $\gamma_{r 2}$ (Fig. 3d) qui valent :

$\gamma_{\mathrm{r} 1}=\frac{\sigma_{\mathrm{c}}}{\mathrm{h}_{\mathrm{r}}}$ (remblai situé à la verticale des colonnes),

$\gamma_{\mathrm{r} 2}=\frac{\sigma_{\mathrm{s}}}{\mathrm{h}_{\mathrm{r}}}$ (remblai situé à la verticale du sol mou).

Pour la détermination de $\gamma_{\mathrm{r} 1}$ et de $\gamma_{\mathrm{r} 2}$, les valeurs de $\sigma$ et de $\sigma_{s}$ sont aussi calculées suivant les étapes a) et b) exposées dans l'étude comparative du paragraphe 2.3.2.

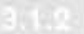

Modélisation des colonnes

(milieu bidimensionnel équivalent)

Le caractère tridimensionnel du milieu renforcé du modèle initial (Fig. 3a) et du modèle réel (Fig. 3d) nous conduit à assimiler les colonnes à des « murs » verticaux équivalents par mètre linéaire de sol (Van Impe et De Beer, 1983). Ceci revient à chercher une épaisseur e du mur équivalent égale à la section réelle $\mathrm{A}_{c}$ de la colonne (Fig. 5a) divisée par l'espacement d entre deux colonnes consécutives (Fig. 5b).

TABLEAUI Construction des trois modèles: sols en présence et paramètres de calcul.

\begin{tabular}{|c|c|c|c|}
\hline Sols Modeles & Modele initial & Homogénéisation de Priebe & $\begin{array}{l}\text { Modèle réel } \\
\text { (état final de report de charge) }\end{array}$ \\
\hline Remblai & \multicolumn{2}{|c|}{$\gamma_{r}\left(c_{r}=0\right), \varphi_{r}$} & $\begin{array}{l}\text { dans la modélisation: } \\
\text { - à la verticale des colonnes: } \gamma_{r+r},\left(c_{r}=0\right), \varphi_{t} \\
\text { - à la verticale du sol: } \gamma_{r e}\left(c_{r}=0\right), \varphi_{r}\end{array}$ \\
\hline Sol mou & $\gamma_{n}, c_{\mathrm{ve}}$ & \multirow{2}{*}{$\begin{array}{l}\text { Milieu homogène équivalent: } \\
\gamma_{e}, c_{e}, \varphi_{e}\end{array}$} & $\gamma_{\mathrm{s}} \cdot \mathrm{cc}_{\mathrm{u}}=\mathrm{c}_{\mathrm{up}}$ \\
\hline Colonnes ballastées & $\gamma_{c}\left(c_{c}=0\right), \varphi_{c}$ & & $\gamma_{c}\left(c_{c}=, 0\right), \varphi_{c}^{\prime}$ \\
\hline
\end{tabular}




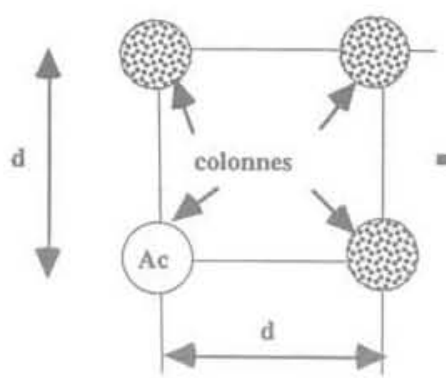

a

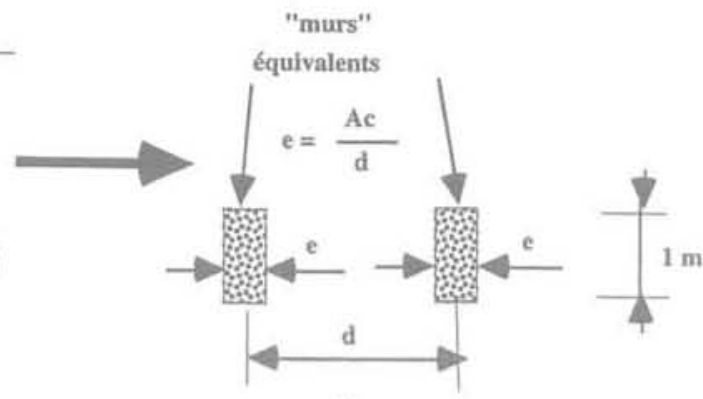

b

FIG,5 Modélisation des colonnes (milieu bidimensionnel équivalent). Column modelling (equivalent two-dimensional medium).

\section{3}

"Taldt " : procédure automatique de construction des modèles et de génération des données

Le modèle initial et le modèle réel nécessitent l'introduction d'une géométrie relativement lourde à traiter sur ordinateur par «Talren » (modélisation du remblai comportant un ou deux sols, des colonnes et du sol). Afin de résoudre cette difficulté, nous avons mis au point un logiciel automatique externe intitulé ( $T$ Taldt » permettant de générer une dizaine de fichiers de données, par minute, avec un modèle comportant 5 à 20 colonnes (Fig. 6).

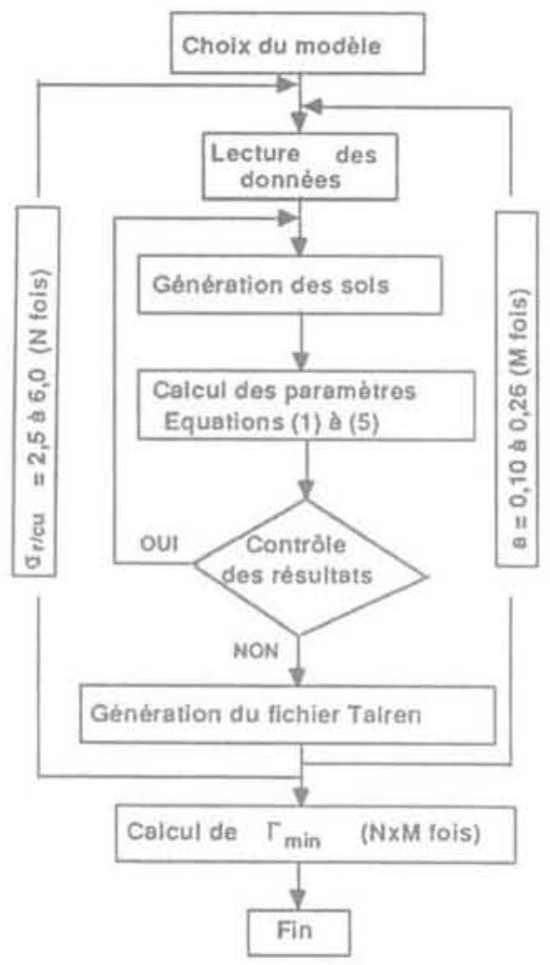

FIG 6 Organigramme de $($ Taldt $)$. "Taldt " flow chart.

\section{2}

\section{Étude comparative et analyse critique des trois modèles de calcul}

La figure 7 présente l'évolution du coefficient de sécurité théorique $\Gamma_{\min }$ exprimé en fonction du pour-

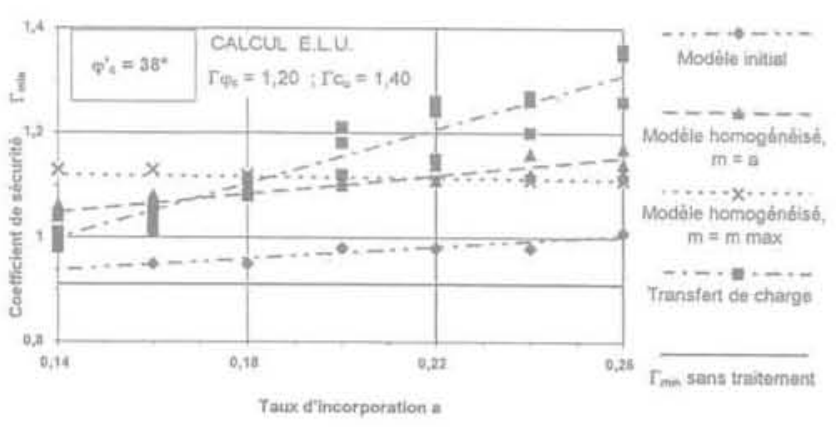

FiG.7 Coefficient de sécurité en fonction de a. Comparaison des trois modèles $\left(\sigma / c_{u}=4\right)$. Minimum safety factors for various modeis.

centage d'incorporation a (compris entre 0,14 et 0,26) pour $\sigma / c_{\text {de }}$ d,0. Les calculs sont menés aux ELU avec les mêmes coefficients de sécurité partiels précédents $\Gamma_{c u}$ de 1,40, $\Gamma_{\phi c}$ et $\Gamma_{\text {or }}$ de 1,20 (ballast et remblai courants) et un coefficient de méthode (Bishop) $\Gamma_{53}$ de 1,125 (Recommandations CLOUTERRE, 1991).

On remarque que les coefficients de sécurité obtenus pour le modèle initial sont les plus faibles et ne présentent qu'une légère augmentation lorsque a croît. Ce résultat concorde avec l'allure linéaire de l'évolution de $\Gamma_{\min }$ en fonction de a pour l'homogénéisation de Priebe à court terme ( $m=a$; Mitchell, 1981). Cette évolution ne diffère que de $10 \%$ près de celle du modèle initial. L'homogénéisation de Priebe, avec $m=m_{\max }$ recommandé par Mitchell (1981) pour les calculs à long terme, conduit à une faible augmentation puis à une légère diminution de $\Gamma_{\min }$

Comparé à ces deux modèles, le modèle réel conduit à une évolution de $\Gamma_{\text {min }}$ nettement plus marquée lorsque a augmente. Les valeurs de $\Gamma_{\min }$ varient en effet de $1,0(\mathrm{a}=0,14)$ à $1,30(\mathrm{a}=0,26)$, soit une amélioration $\Gamma_{\min }$ de l'ordre de $30 \%$ pour une augmentation de a de $85 \%$. A conditions de stabilité identiques $\left(\Gamma_{\text {min }}=1,10\right.$ par exemple) et avec les traitements usuels ( $\mathrm{a}=0,14$ à 0,22$)$, le calcul basé sur le modèle initial conduit à un surdimensionnement de plus de $50 \%$, la procédure d'homogénéisation à au moins $15 \%$. Le choix d'un coefficient multiplicateur k (équation 3b) supérieur à 4 (de 6 par exemple, Nahrgang, 1976) ne pourrait qu'accentuer cette différence.

La présente étude comparative montre que le modèle initial n'apporte qu'une faible amélioration de la résistance au cisaillement du sol à l'aide des colonnes frottantes. Ce modèle peut s'appliquer au calcul à court terme dans l'hypothèse où le remblai serait mis sur 
toute la hauteur instantanément. L'homogénéisation de Priebe définie pour $\mathrm{m}=\mathrm{m}_{\max }$ montre que $\Gamma_{\min }$ diminue lorsque a augmente jusqu'a une valeur caractéristique $a_{c}$ à partir de laquelle le traitement devient a priori inutile pour améliorer la stabilité. Ce résultat, étonnant à première vue, s'explique par le fait que l'homogénéisation de Priebe aboutit, pour a élevé, à un sol d'assise homogénéisé assez frottant et peu cohérent. Pour ce type de matériau, les règles et les analyses classiques à la rupture conduisent à des surfaces de rupture peu profondes qui peuvent ne pas correspondre à la réalité des phénomènes de glissement (Dhouib et al., 1993). La prise en compte du report de charge conduit, comme le modèle initial, à un modèle géométrique délicat à traiter sur ordinateur. A cela s'ajoute la nécessité de déterminer les contraintes à la base du remblai. On peut recourir à une procédure numérique de calcul par éléments finis (Liausu et Juillié, 1990) ou à une approche basée sur l'interaction sol-structure (Gilbert, 1995). Pour le renforcement de la vase de Tunis par des colonnes de sables, Bouassida (1996) a réalisé une étude expérimentale qui permet de valider la résistance en compression théorique d'une cellule composite confinée.

En dépit de ces difficultés, la prise en compte de l'état final de report de charge se traduit par une nette augmentation de $\Gamma_{\min }$ lorsque a croît et permet d'appréhender, comme nous allons le montrer dans ce qui suit, l'influence de la variation des paramètres de dimensionnement sur l'évolution du coefficient de sécurité $\Gamma_{\text {min }}$
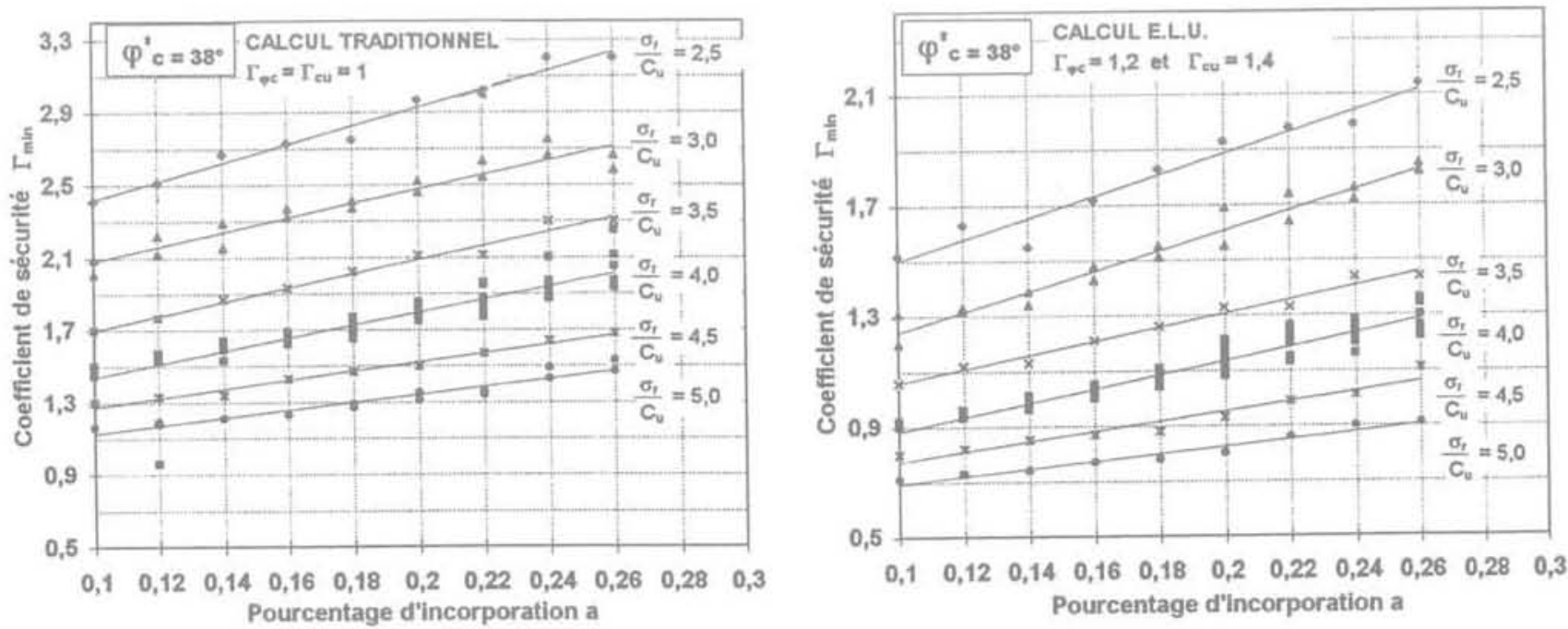

a - $\varphi_{c}^{\prime}=38^{\circ}$
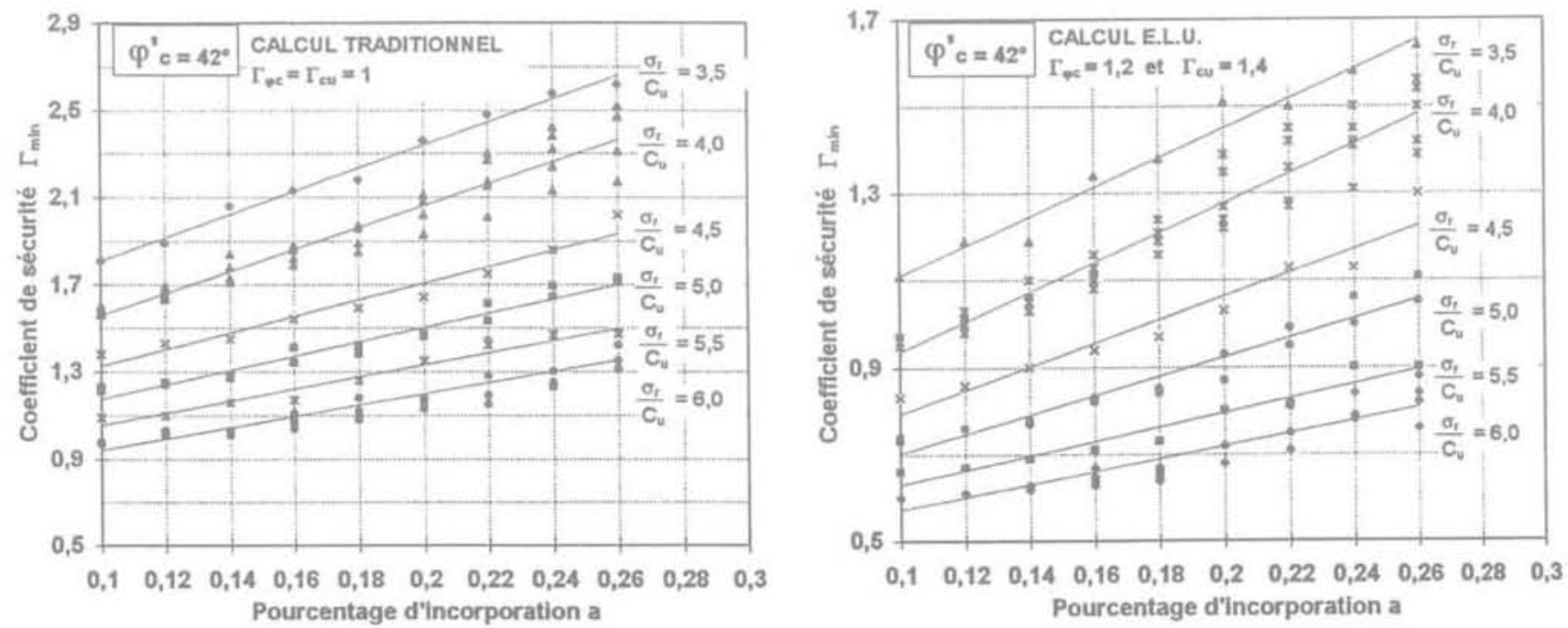

b - $\varphi_{c}{ }^{\prime}=42^{\circ}$.

FiG.8 Coefficient de sécurité $\Gamma_{\min }$ en fonction de a pour divers taux de chargement $\sigma_{\mathrm{r}} / c_{u}$ (calcul aux ELU et calcul traditionnel).

Minimum safety factors $\Gamma_{\min }$ versus area ratio a for various loading levels $\sigma / c_{u}$

a. $\varphi^{\prime}=38^{\circ}$.

b. $\varphi_{c}^{\prime}=42^{\circ}$. 


\section{Étude de stabilité : influence de la variation des paramètres}

4.1

\section{Conditions de stabilité en fonction du volume traité}

L'évolution du coefficient de sécurité $\Gamma_{\text {min }}$ en fonction du pourcentage de substitution a est presentée sur la figure $8 \mathrm{a}$ pour $\varphi^{\prime}$ de $38^{\circ}$ et $8 \mathrm{~b}$ pour $\varphi^{\prime}$ de $42^{\circ}$ et pour des taux de chargement $\sigma / \mathrm{c}$ allant de 2,5 à 6,0 (hauteurs de remblais de 3,0 à $12,0 \mathrm{~m}$ et $c_{4}$ de 15 à $50 \mathrm{kPa}$ ). L'examen des courbes de la figure 8 montre que $\Gamma_{\text {. }}$ présente une variation moyenne quasi linéaire en fonction de a. Cette variation croit nettement avec a, ce qui montre l'efficacité du traitement. Lorsque $\sigma / c_{u}$ augmente, le coefficient de sécurité diminue et varie peu pour des valeurs croissantes de a. L'augmentation de l'angle de frottement interne $\varphi^{\prime}$ de $38^{\circ}$ à $42^{\circ}$ conduit à une amélioration de $\Gamma_{\text {an }}$ d'environ $5 \%(\mathrm{a}=0,10)$ à $15 / 20 \%(a=0,26)$; ce qui montre que le choix d'un matériau de traitement plus frottant ne peut être intéressant que pour des volumes de traitement importants.

\section{Incidence des caractéristiques de cisaillement du remblai et des colonnes}

On a considéré un matériau de remblai sans cohésion $\left(c_{r}=0\right)$ et avec différentes valeurs courantes de l'angle de frottement interne $\left(\varphi_{r}=25,30\right.$ et $\left.35^{\circ}\right)$. L'évolution de $\Gamma_{\text {min }}$ en fonction du taux d'incorporation a est irrégulière. En plus, l'amélioration de $\Gamma_{\text {min }}$ reste faible et ne dépasse guère les $5 \%$ quel que soit le volume de substitution. Cette amélioration est plus faible en particulier pour $\sigma / c_{u}$ plus élevé. Ce résultat s'explique par le fait que les coefficients de sécurité $\Gamma_{\text {min }}$ calculés correspondent à des cercles de rupture théoriques relativement profonds. Ces cercles traversent le remblai d'une façon presque subverticale, conduisant à une contrainte normale et à une résistance au cisaillement faibles. A cela s'ajoute une hypothèse inhérente au logiciel « Talren » et restrictive, même si elle peut correspondre à un phénomène bien réel en cas d'apparition de fissures en surface du remblai. Cette hypothèse consiste à annuler la contrainte normale au droit de la zone subverticale de la surface potentielle de rupture.

L'influence de la cohésion dans la colonne a été étudiée pour un remblai courant $\left(\varphi=30^{\circ}\right)$ et sans cohésion $\left(c_{t}=0\right)$ ). Les colonnes sont considérées à la fois frottantes avec $\varphi_{\mathrm{c}}=30^{\circ}$ et cohérentes avec $c_{\mathrm{c}}=0$ à $20 \mathrm{kPa}$ (cas par exemple d'un matériau sableux avec une fraction de ciment). Il ressort de l'étude que la variation de $\Gamma_{\min }$ en fonction de a est également irrégulière. L'augmentation de a est accompagnée d'une amélioration de $\Gamma_{\min }$ de 5 à $10 \%$. Cependant, cette amélioration est moins importante pour $\sigma / c_{u}$ plus élevé. Ce résultat s'explique par le fait que lorsque $\sigma / c_{i}$ croit, le taux de contribution $\left(\mathrm{m}_{\mathrm{c}}\right)$ de la colonne diminue.

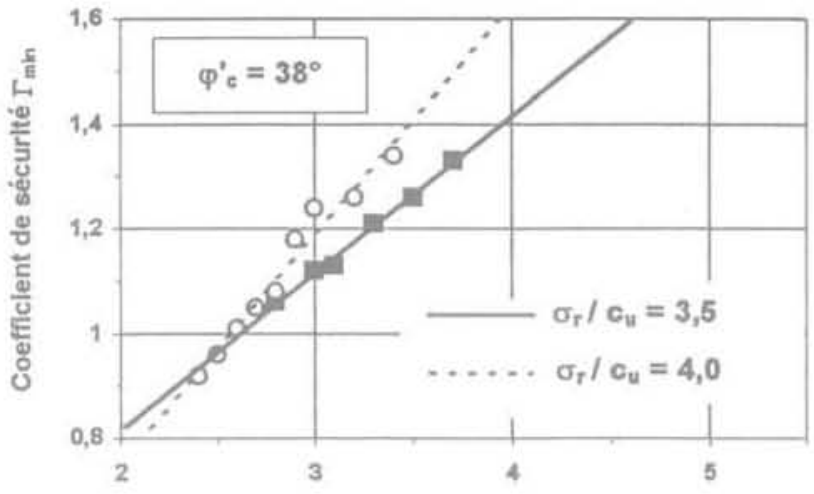

Rapport de concentration des contraintes $\mathbf{n}$

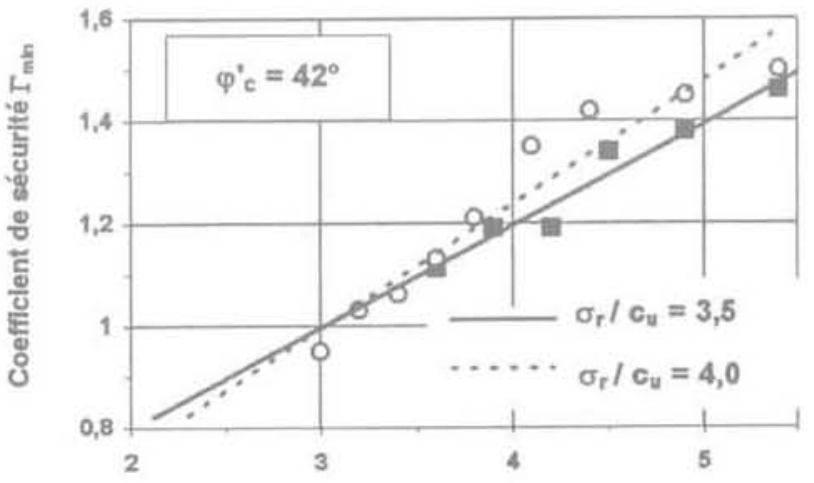

Rapport de concentration des contraintes $\mathbf{n}$

FIG. 9 Incidence du rapport de concentration des contraintes n sur $\Gamma_{\text {min }}$

Minimum safety factors : effect of stress concentration ratio $n$. 

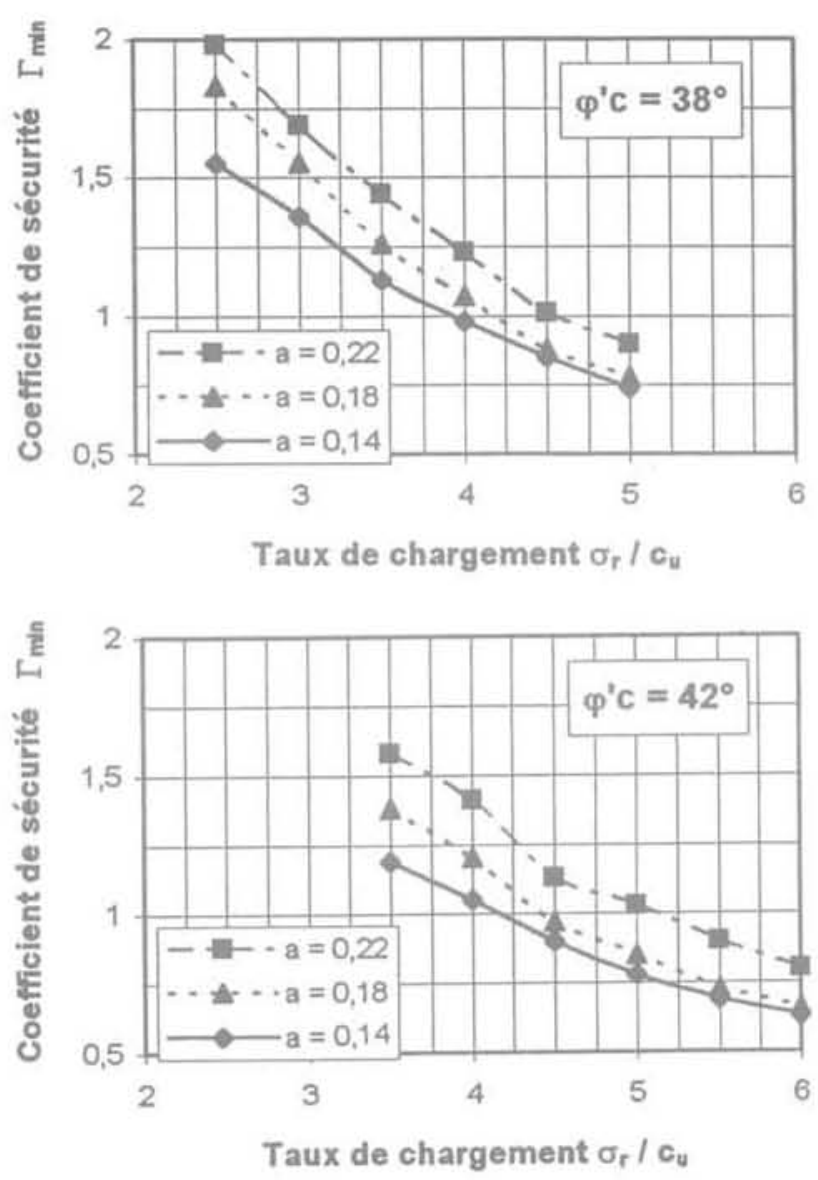

FIG. 10 Incidence du taux de chargement $\sigma_{\mathrm{v}} / \mathrm{c}_{\mathrm{u}}$ sur les valeurs de $\Gamma_{\min }$

Minimum safety factors ; effect of loading level.

croit. Cette diminution est d'autant plus marquée que le taux de chargement est faible. Ce résuitat concorde avec les variations de $\Gamma_{\min }$ en fonction de a des graphiques de la figure 8 et s'explique par le fait que le taux de contribution de la colonne décroit lorsque $\sigma / c_{u}$ croit. L'augmentation de l'angle de frottement interne du ballast $\varphi^{\prime}$, de $38^{\circ}$ (Fig. 9a) et $42^{\circ}$ (Fig. 9b) n'apporte d'amélioration plus marquée que pour les taux d'incorporation a plus élevés.

\section{Conclusion}

L'analyse menée montre que la modélisation basée sur le transfert de charge conduit à une meilleure prise en compte de la contribution du ballast du fait de l'amélioration de la résistance au cisaillement au sein de la colonne. Ce qui se traduit par une augmentation du coefficient de sécurité global notamment dans les plages de traitement couramment utilisées (14 à 20\%). Par contre, les calculs de stabilité faisant appel au modèle initial montrent que l'amélioration de la stabilité reste faible et qu'ils doivent être limités à l'analyse à très court terme. Ces deux procédures de modélisation nécessitent l'introduction d'une géométrie relativement lourde sur ordinateur (modélisation du remblai, des colonnes et du sol). Pour résoudre cette difficulté, nous avons mis au point un logiciel externe " Taldt » qui génère de façon automatique les données de calcul propres à « Talren ».

L'homogénéisation de type Priebe offre une modélisation très simple à traiter sur ordinateur puisqu'elle conduit uniquement à deux milieux distincts (remblai et sol sous-jacent homogénéisé). Cependant, elle aboutit à une stabilisation, voire à une chute du coefficient de sécurité lorsqu'on augmente le taux d'incorporation. Cette chute s'explique par le fait que l'homogénéisation aboutit, pour a élevé, à un sol homogénéisé assez frottant et peu cohérent où les règles et les analyses classiques à la rupture conduisent à des surfaces de rupture peu profondes qui peuvent ne pas correspondre à la réalité des phénomènes (Dhouib et al., 1993).

La présente étude montre aussi que l'amélioration de la stabilité est faible lorsque l'on augmente l'angle de frottement du matériau du remblai. Ce résultat est dû à la faible contrainte normale mobilisée en tête des cercles de rupture relativement très profonds et subverticaux au sein du remblai. Par contre, la stabilité est légèrement améliorée lorsque le matériau de traitement est cohérent (cas du sable-ciment ou sable avec une fraction de fines). Le report de charge conduit, par le biais de la concentration des contraintes sur les colonnes, à l'amélioration de la stabilité. Par contre, l'augmentation du taux de chargement fait chuter le coefficient de sécurité.

Il $y$ a lieu de noter enfin qu'il s'agit là d'une étude théorique basée sur une analyse à la rupture classique et dont la validation expérimentale est difficile à mettre en œuvre,

\section{$\overline{\text { Bibliographie }}$}

Aboshi H. Ichimoto E., Enoki M.. Harada K. - The Compozer, a method to improve characteristics of soft clays by inclusion of large diameter sand columns. Colloque international Renforcement des sols : Terre Armée et autres méthodes, Paris. Presses des Ponts, vol. 1, p. 211-216, 1979.

Balaam N.P., Poulos H.G. - The behaviour of foundations supported by clays stabi- lized by stone columns. Proc. 8th EC on SMFE Heisinki, vol. 1, p. 199-204, 1983. Balaam N.P., Poulos H.G., Brown P.T. Settlement analysis of soft clays reinforced with granular piles. University of Sydney, School of Civil Engineering. Research Report R 305.

Blondeau F., Christiansen M., Guilloux A. Schlosser F. - $\alpha$ Talren $»$ : méthode de calcul des ouvrages en sols renforcés.
Colloque international Renforcement des sols en place, Paris. Presses des Ponts, p. 219-224, 1984.

Bouassida M - Étude expérimentale du renforcement de la vase de Tunis par colonnes de sables: application pour la validation de la résistance en compression théorique d'une cellule composite confinée. Revue Francaise de Géotechnique, $n^{\circ} 75$, p. 3-12, 1996. 
Brauns J. - Die Anfangstraglast von Schottersaûlen im bindingen Unterground. Die Bautechnik, vol. 55, n'8, p.263-271, 1978.

De Buhan P., Salençon J. - Analyse de la stabilitė des ouvrages en sols renforcés par une méthode d'homogẻnéisation. Revue Française de Géotechnique, $n^{\circ} 41$. p. 29-43, 1987.

Dhouib A. Soyez B., Shahrour I. Réflexions sur les problèmes de calcul et de dimensionnement des colonnes ballastées sous chargement monotone. Colloque franco-polonais de Mécanique des sols. Douai, p. 305-312, 1993.

Dhouib A., Gambin M., Soyez B. - Incidence du mécanisme de transfert de charge sur la stabilité des remblais d'ouvrages en terre sur sols compressibles traités par réseau de colonnes ballastées. Third International Conference On Ground Improvement GeosystemsDensification and Reinforcement. London, Thomas Telford, 1997

Dhouib A., Stoehr B. - Techniques d'amélioration des sols par vibration profonde: origine, développement et méthodes de dimensionnement. «Entretiens de la Technologie », 1997. écition européenne, École centrale de Lifle. Les Dossiers des technologies, Ed. Londez Conseil, p. 271-277, 1997.

DTU (Documents techniques unifiés) 13-2. - Colonnes Ballastées, chap. III, 1978

Gilbert C. - Une nouvelle approche des calculs d'interaction sol-structure. Revue Française de Géotechnique, $n^{\circ} 72$, p. 3-9, 1995 .

Goughnour R.R., Bayuk A.A. - Analysis of stone column-soil matrix interaction under vertical load, Colloque international Renforcement des sols: Terre Armée et autres méthodes, Paris. Press des Ponts, vol. 1, p. 271-277, 1979.

Ghionna V., Jamiolkowski M. - Colonne di ghiaia. X Ciclo di conferenze dedi- cate ai problemi di meccanica dei terreni e ingegneria delle fondazion metodi di miglioramento dei terreni. Politecnico di Torino Ingegneria, atti dell'istituto di scienza delle construzioni, $n^{\circ} 507,1981$

Greenwood D.A. - Mechanical improve. ment of soils below ground surface. Conf. on Ground Engineering. Institution of Civil Engineers, Londres, paper II, p. 11-22, 1970

Hughes J.M.O., Withers N.J. - Reinforcing of soft cohesive soils with stone columns. Ground Engineering, vol. 7. $n^{\circ} 3, \mathrm{p}, 42-49,1974$

Iorio J.P. Matichard Y., Soyez B., Besançon G. - Etude du comportement d'une culée en Terre Armée fandée sur colonnes ballastées. Coll. Interaction solstructures, Paris. Presses des Ponts, 1987.

Liausu Ph., Juillié Y. - Procédures de contrôles sur un chantier de plots ballastées, Voies ferrẻes ELA 3 à Kourou (Guyane française). Revue Travaux, $n^{\circ} 655,1990$.

Nahrgang E. - Untersuchung des Tragverhaltens von eingrüttelten Schottersäulen an Hand von Moldellversuchen. Baumaschine und Bautechnik, vol. 23 $n^{\circ} 8$, p. 391-404, 1976

Meiketsu Enoki, Norio Yagi, Ryuich Yatabe, Eisamburo Ichimoto - Shearing characteristic of composite ground and its application to stability analysis. Deep Foundation improvements: Design, Construction and Testing, ASTM STP 1089. Malvin 1. Esrig and Robert C. Bachus, Eds, American Society for Testing and Materials, Philadelphia, p. 1931, 1991.

Mitchell J.K. - Soil improvement. State of the art report. $10^{\circ}$ Cong. int. Mécanique des sols et des travaux de fondations, Stockholm, vol. 4, p. $509-565,1981$.

Priebe H. - Abschätzung des Setzungverhaltes eines durch Stopfverdichtung verbesserten Baugrundes. Die Bautechnik, vol. 53, n० 5, p. 160-162, 1976.

Priebe H. - Abschätzung des Scherwiderstandes eines durch Stopfverdichtung verbesserten Baugrundes. Die Bautechnik, vol. 55, n9, p. 281-284, 1978.

Priebe H. - Vibro replacement. Design Criteria and Quality Control. Deep Foundation improvements : Design, Construction and Testing, ASTM STP 1089, Malyin I. Esrig and Robert C. Bachus, Eds, American Society for Tes. ting and Materials, Philadelphia, p. 62. 72, 1991.

Pruchnicki E., Shahrour I. - Application de la théorie de Thomogénéisation aux colonnes ballastées. Annales de ITTBTP. $n^{\circ} 496, p, 117-128,1991$.

Recommandations CLOUTERRE - Paris, Presse des Ponts, 268 p. 1991.

Schulze G. - Consolidation des sols par vibration profonde et par compactage dynamique. Sêminaire sur la consolida. tion des sols et des roches in situ dans le gênie civil, Stresa, p. 83-115, 1978

Soyez B. - Méthodes de dimensionnement des colonnes ballastées. Bulletin de Liaison des LPC, $\mathrm{n}^{\circ} 135$, p. 35-51, 1985

Soyez B, Magnan J.-P. Delfaut A. - Loading tests on a clayey hydraulic fill stabilized by lime-treated soil columns. Proc. 8th European Conf. on SMFE, Helsinki, vol. 1, p. 951-954, 1983.

Smoltczyk U. - Deep compaction-general report. 8th European Conf. on SMFE. Helsinki. Speciality Session 3, vol. 3, p. 1105-1116, 1983.

Van Impe W., de Beer E. - Improvement of settlement behaviour of soft layers by means of stones columns. 8th European Conference on SMFE, Heisinki, vol. 1 . p. 309-312, 1983.

Vautrain J. - Comportement et dimensionnement des colonnes ballastées. Revue Française de Géotechnique, $n^{\circ} 11$, p. 59 . 73, 1980, 